\title{
Correction to: A Framework for Image Dark Data Assessment
}

\author{
Yu Liu, Yangtao Wang, Ke Zhou, Yujuan Yang, \\ Yifei Liu, Jingkuan Song, and Zhili Xiao
}

\section{Correction to: \\ Chapter "A Framework for Image Dark Data Assessment" in: J. Shao et al. (Eds.): Web and Big Data, LNCS 11641, https://doi.org/10.1007/978-3-030-26072-9_1}

The original version of the chapter "A Framework for Image Dark Data Assessment", starting on p. 3 was not correct. The abstract section and the keywords have been exchanged. This have been now corrected.

The correct abstract and keywords as follows:

\begin{abstract}
Blindly applying data mining techniques on image dark data whose content and value are not clear, is highly likely to bring undesired result. Therefore, we propose an assessment framework which includes offline and online stages for image dark data. In offline stage, we first transform images into hash codes by Deep Self-taught Hashing (DSTH) algorithm, then construct a semantic graph, and finally use our designed Semantic Hash Ranking (SHR) algorithm to calculate the importance score. During online stage, we first translate the user's query into hash codes, then match the suitable data contained in the dark data, and finally return the weighted average value of these matched data to help the user cognize the dark data. The results on real-world dataset show our framework can apply to large-scale datasets, help the user conduct subsequent data mining work.
\end{abstract}

Keywords: Image dark data • Deep self-taught hashing • Semantic hash ranking $\cdot$ Assessment

The updated version of this chapter can be found at https://doi.org/10.1007/978-3-030-26072-9_1 